\title{
Somebody's Poisoned the Waterhole: ASPCA Poison Control Center Data to Identify Animal Health Risks
}

\author{
Kristen Alldredge ${ }^{\star 1,3}$, Leah Estberg ${ }^{1}$, Cynthia Johnson ${ }^{1}$, Howard Burkom ${ }^{2}$ and Judy \\ Akkina $^{1}$
}

${ }^{1}$ USDA-APHIS-VS, Ft Collins, CO, USA; ${ }^{2}$ Johns Hopkins University Applied Physics Laboratory, Laurel, MD, USA; ${ }^{3}$ UC Davis, Davis, CA, USA

\begin{abstract}
Objective
To describe the value of the American Society for the Prevention of Cruelty to Animals (ASPCA) Poison Control Center (APCC) livestock animal calls as a passive data stream for biosurveillance of number of calls, species affected, toxicant exposures, and clinical syndromes.
\end{abstract}

\section{Introduction}

The APCC hotline fields daily calls regarding potential animal intoxications from the US, its territories, and Canada. We explored the value of these data for identifying increased occurrences of intoxications related to livestock and poultry species, toxicant product categories, clinical syndromes, and illness severity. These data proved valuable for identifying risks of toxicant exposures by species, product category, and season. In addition to identifying intoxication risks to animal health, these data could be used to monitor for infectious outbreaks that may initially be confused for intoxications.

\section{Methods}

The APCC hotline was contracted to provide ongoing de-identified call data from calls starting 01 October 2013 that were related to the following livestock taxa: equine, bovine, caprine, porcine, poultry, ovine, and camelid. The ingredient lists provided were categorized into mutually exclusive product categories (e.g. pesticide, prescription medication). Clinical signs were categorized into nonmutually exclusive syndromic categories (e.g. death, respiratory). Illness severity was categorized as none, mild/moderate, severe, and death. We used the Early Aberration Reporting System (EARS) C3 algorithm to identify counts (signals) for weekly toxicosis, species and syndromic events that occurred at a frequency more than three standard deviations above the expected occurrence.

\section{Results}

On average, the APCC hotline took 9 calls a week regarding our species of interest (range: 1-22). Calls came from all 50 states, Puerto Rico, and seven Canadian provinces. Pesticides were the most frequent intoxication product (36.2\% of exposures) and were primarily involved in calls regarding horses or cattle. Calls regarding pigs were more likely to involve exposure to a human product (e.g. human medications, human food), likely reflecting that most pigrelated calls were about companion animal breeds (e.g. pot-bellied pigs). Horses were the most common species discussed. Most animals involved in calls did not have clinical signs. Of those that did show specific clinical signs, most were gastrointestinal or dermatological. Death was most frequently associated with pesticide intoxication. Pesticide intoxication had a markedly seasonal trend, with peaks in pesticide calls seen May-August. Figures 1 and 2 show the $\mathrm{C} 3$ model for detecting increased incidence of calls about pesticides and select species/syndromes respectively.

\section{Conclusions}

Analyzing APCC calls related to livestock and poultry species is useful for the purpose of risk identification and animal health monitoring. Identification of seasonal trends - such as those with pesticides - can inform policymakers and livestock owners of the increased risk during specific times of year. Targeted education preceding summer months could help reduce the risk of pesticide exposure to animals. Identifying peaks in specific clinical signs could help identify unusual health trends. Use of these types of passive data streams provides a valuable information source for all health professionals interested in biosurveillance, animal health management, and risk identification.
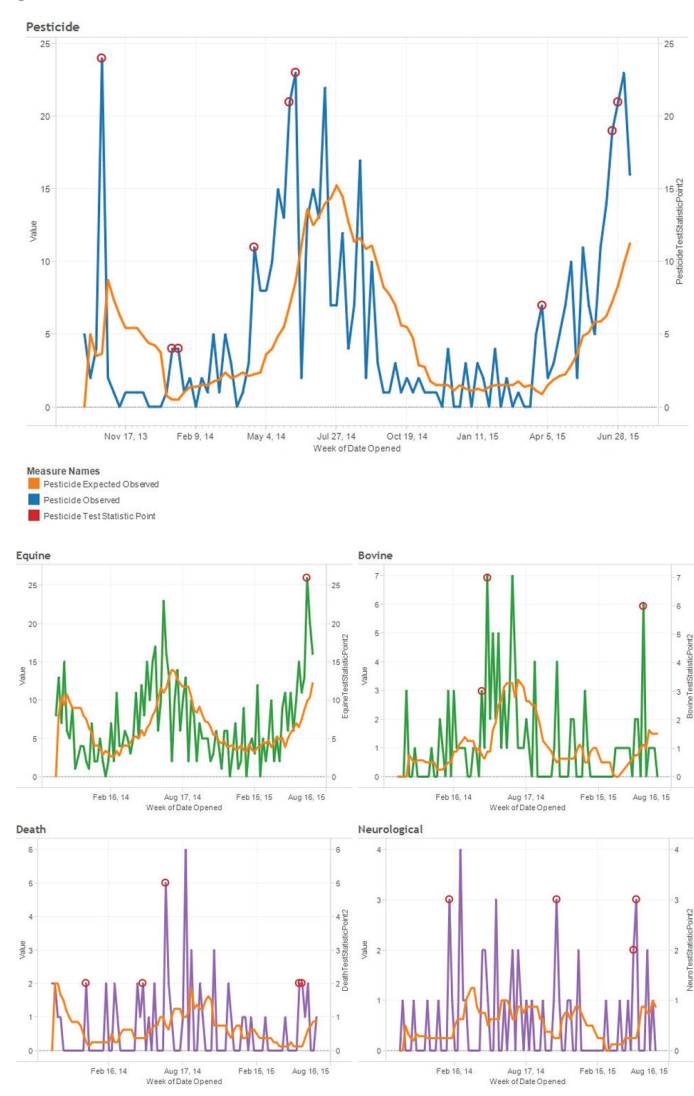

Keywords

Risk identification; Passive surveillance; Biosurveillance; Animal health monitoring; Toxicoses

\section{Acknowledgments}

This work supported by USDA-APHIS-VS-CEAH and the Saul T. Wilson, Jr., Scholarship. Copyright@ 2015 . The American Society for the Prevention of Cruelty to Animals (ASPCA). All Rights Reserved.

\section{${ }^{*}$ Kristen Alldredge}

E-mail: Kristen.L.Alldredge@aphis.usda.gov 